\title{
Elastic Properties of Carbon Nanotubes
}

\author{
Qiang Han and Hao Xin \\ School of Civil Engineering and Transportation \\ South China University of Technology \\ Guangzhou \\ People's Republic of China
}

\section{Introduction}

The geometric structure of carbon nanotubes (CNTs) can be considered to be the curling of graphene (graphite sheet) (Gao \& Li, 2003; Shen, 2004). The physical parameters of carbon in graphite are widely adopted in the molecule dynamics (MD) simulations or other theoretical studies of CNTs, such as the bond energy and length of C-C, the bond angle of C-C-C, etc (Belytschko et al., 2002; Mayo et al., 1990; Xin et al., 2007, 2008). Previous researches have shown that the elastic modulus of small CNTs changes a lot when the radius varies. However, the elastic modulus of CNTs fairly approaches that of graphene, while the radius of CNTs is large enough. Therefore, it is necessary to have a clear understanding of the mechanical properties of graphene for the further realization of the properties of CNTs.

Numerous researchers carried out experiments to measure the effective elastic modulus of CNTs (Krishnan et al.,1998; Poncharal et al., 1999). They reported the effective Young's modulus of CNTs ranging from 0.1 to $1.7 \mathrm{TPa}$, decreasing as the diameter increased, and the average was about 1.0 1.2 TPa. MD simulations have provided abundant results for the understanding of the buckling behavior of CNTs. The Young's modulus of the CNTs was predicted about 1.0 1.2 TPa through various MD methods (Jin \& Yuan, 2003; Li \& Chou, 2003; Lu, 1997). Hu et al. (Hu et al., 2007) proposed an improved molecular structural mechanics method for the buckling analysis of CNTs, based on Li and Chou's model (Hwang et al., 2010) and Tersoff-Brenner potential (Brenner, 1990). Due to the different methods employed on various CNTs in these researches, the reported data scattered around an average of $1.0 \mathrm{TPa}$.

The elastic properties were also discussed in the theoretical analysis by Govinjee and Sackman (Govinjee and Sackman, 1999) based on Euler beam theory, which showed the size dependency of the elastic properties at the nanoscale, which does not occur at continuum scale. Harik (Harik, 2001) further proposed three non-dimensional parameters to validate the beam assumption, and the results showed that the beam model is only proper for CNTs with small radius. Liu et al (Liu et al., 2001) reported the decrease of the elastic modulus of CNTs with increase in the tube diameter. Shell model was also used in some researches (Wang et al., 2003), to study axially compressed buckling of multi-walled CNTs. And studies by Sudak (Sudak, 2003) reported that the scale effect of CNTs should not be ignored. Wang et al (Wang et al., 2006) investigated the buckling of CNTs and the results showed that the critical buckling load drawn with the classical continuum theory is higher than that 
with considering the scale effect. There are some other researches also reporting clear scale effect on the vibration of CNTs (Wang \& Varadan, 2007; Zhang et al., 2005). Li and Chou (Li \& Chou, 2003) put forward a truss model for CNTs and their studies showed the radiusdependence of elastic modulus of SWCNTs. Finite element analysis (FEA) is also employed in researches on the mechanical properties of CNTs (Yao \& Han, 2007, 2008; Yao et al., 2008). An equivalent model is established in this chapter, based on the basic principles of the anisotropic elasticity and composite mechanics, for the analysis of the elastic properties of graphite sheet at the nanometer scale. With this equivalent model, the relationship between the nanotube structure and the graphite sheet is built up, and the radial scale effect of the elastic properties of CNTs is investigated.

\section{Constitutive equations of orthotropic system}

The essential difference between the basic equations of anisotropic materials and those of isotropic ones is in their constitutive equations, which means the usage of the anisotropy Hooke law for the anisotropic constitutive equation and the isotropy one for the other. The phenomenon reflected from the anisotropy equations is more accurate than from the isotropy ones, though this distinction also makes the calculation with the anisotropy equations much more complicated.

One of the anisotropic systems, with three mutually perpendicular principal axes of elasticity, is called an orthotropic system (Fig. 1). If the three principal axes of elasticity are defined as $x_{1}, x_{2}$ and $x_{3}$, the constitutive equation of orthotropic system can be obtained as follow,
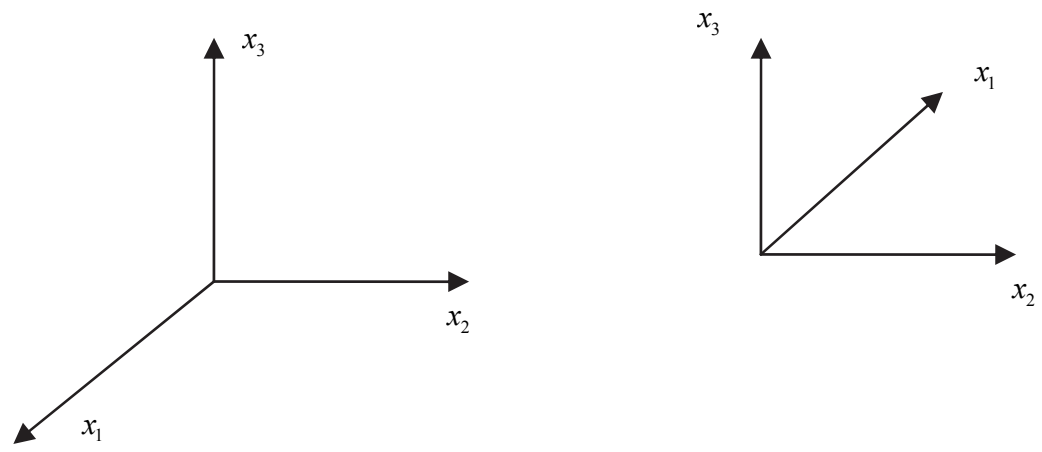

Fig. 1. The principal elastic axes of orthotropic system: a) right-hand coordinate system; b) left-hand coordinate system

$$
\left\{\begin{array}{l}
\varepsilon_{1} \\
\varepsilon_{2} \\
\varepsilon_{3} \\
\varepsilon_{4} \\
\varepsilon_{5} \\
\varepsilon_{6}
\end{array}\right\}=\left[\begin{array}{llllll}
S_{11} & S_{12} & S_{13} & & & \\
S_{12} & S_{22} & S_{23} & & & \\
S_{13} & S_{23} & S_{33} & & & \\
& & & S_{44} & & \\
& & & & S_{55} & \\
& & & & & S_{66}
\end{array}\right]\left\{\begin{array}{l}
\sigma_{1} \\
\sigma_{2} \\
\sigma_{3} \\
\sigma_{4} \\
\sigma_{5} \\
\sigma_{6}
\end{array}\right\}
$$


And the inverse function of the equation (1) is,

$$
\left\{\begin{array}{l}
\sigma_{1} \\
\sigma_{2} \\
\sigma_{3} \\
\tau_{23} \\
\tau_{31} \\
\tau_{12}
\end{array}\right\}=\left[\begin{array}{llllll}
C_{11} & C_{12} & C_{13} & & & \\
C_{12} & C_{22} & C_{23} & & & \\
C_{13} & C_{23} & C_{33} & & & \\
& & & C_{44} & & \\
& & & & C_{55} & \\
& & & & & C_{66}
\end{array}\right]\left\{\begin{array}{c}
\varepsilon_{1} \\
\varepsilon_{2} \\
\varepsilon_{3} \\
\gamma_{23} \\
\gamma_{31} \\
\gamma_{12}
\end{array}\right\}
$$

where,

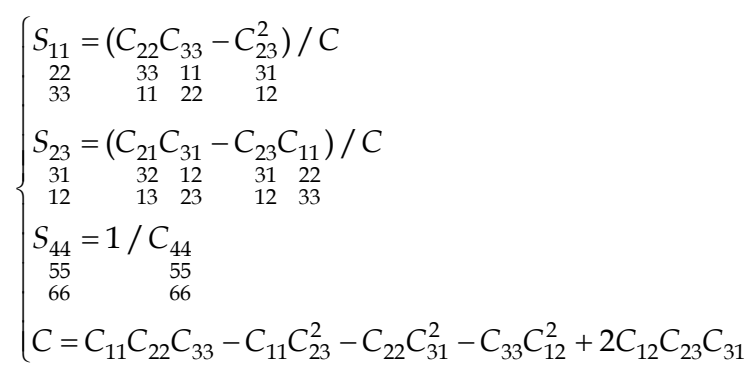

Equation (3) is also correct if we make exchanges of $C$ for $S$ and $S$ for $C$.

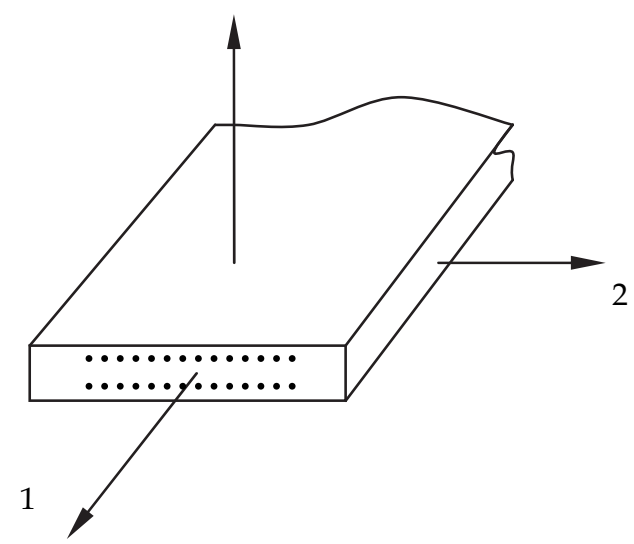

Fig. 2. Sketch of macroscopic homogeneous orthotropic material

The unidirectional fiber composed composite materials can be treated as orthotropic systems, for which the three axes in the right-handed coordinate system in Fig. 2 are the principal material axes and the axis 1 is along the fiber length. Thus, the components of equation (1) can be given minutely in detail, 


$$
\begin{aligned}
& \varepsilon_{1}=S_{11} \sigma_{1}+S_{12} \sigma_{2}+S_{13} \sigma_{3}=\frac{1}{E_{1}} \sigma_{1}-\frac{v_{12}}{E_{2}} \sigma_{2}-\frac{v_{13}}{E_{3}} \sigma_{3} \\
& \varepsilon_{2}=S_{21} \sigma_{1}+S_{22} \sigma_{2}+S_{23} \sigma_{3}=-\frac{v_{21}}{E_{1}} \sigma_{1}+\frac{1}{E_{2}} \sigma_{2}-\frac{v_{23}}{E_{3}} \sigma_{3} \\
& \varepsilon_{3}=S_{31} \sigma_{1}+S_{32} \sigma_{2}+S_{33} \sigma_{3}=-\frac{v_{31}}{E_{1}} \sigma_{1}-\frac{v_{32}}{E_{2}} \sigma_{2}+\frac{1}{E_{3}} \sigma_{3} \\
& \gamma_{23}=S_{44} \tau_{23}=\frac{1}{G_{23}} \tau_{23} \\
& \gamma_{31}=S_{55} \tau_{31}=\frac{1}{G_{31}} \tau_{31} \\
& \gamma_{12}=S_{66} \tau_{12}=\frac{1}{G_{12}} \tau_{12}
\end{aligned}
$$

where,

$$
\left\{\begin{array}{l}
S_{i j}=S_{j i} \\
\frac{v_{i j}}{E_{j}}=\frac{v_{j i}}{E_{i}}
\end{array}\right.
$$

\section{Macro-mechanics fundamental principle of composite material}

\subsection{Constitutive equations of monolayer under plane stress}

The monolayer composite with unidirectional fiber can be considered as a homogeneous orthotropic material in the macro analysis. Fig. 3 displays the three principal material axes and the axis 3 is perpendicular to the mid-plane of the monolayer. Suppose the monolayer is in a plane stress state, there are the in-plane stresses,

$$
\sigma_{1}, \quad \sigma_{2}, \quad \tau_{12}\left(\sigma_{6}\right)
$$

and the out-plane stresses,

$$
\sigma_{3}=\tau_{23}\left(\sigma_{4}\right)=\tau_{13}\left(\sigma_{5}\right)=0
$$

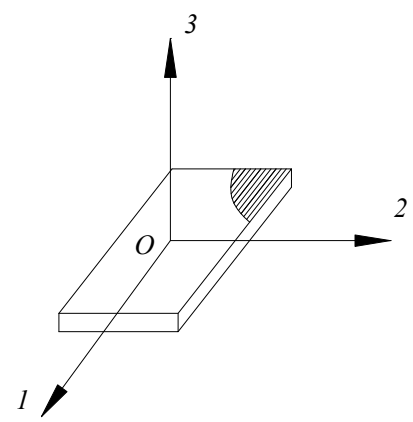

Fig. 3. Sketch of monolayer composite with unidirectional fiber 
Thus, the constitutive equation (1) can be given in two parts as follows,

$$
\begin{aligned}
& \varepsilon_{3}=S_{31} \sigma_{1}+S_{32} \sigma_{2}=-\frac{v_{31}}{E_{1}} \sigma_{1}-\frac{v_{32}}{E_{2}} \sigma_{2} \\
& \gamma_{23}=0, \quad \gamma_{31}=0 \\
& \varepsilon_{1}=S_{11} \sigma_{1}+S_{12} \sigma_{2}=\frac{1}{E_{1}} \sigma_{1}-\frac{v_{12}}{E_{2}} \sigma_{2} \\
& \varepsilon_{2}=S_{21} \sigma_{1}+S_{22} \sigma_{2}=-\frac{v_{21}}{E_{1}} \sigma_{1}+\frac{1}{E_{2}} \sigma_{2} \\
& \gamma_{12}=S_{66} \tau_{12}=\frac{1}{G_{12}} \tau_{12}
\end{aligned}
$$

The equation (8) is for the out-plane strain and the equation (9) for the in-plane strain. And the equation (9) can also be written in a matrix form,

$$
\left\{\begin{array}{l}
\varepsilon_{1} \\
\varepsilon_{2} \\
\gamma_{12}
\end{array}\right\}=\left[\begin{array}{ccc}
S_{11} & S_{12} & 0 \\
S_{21} & S_{22} & 0 \\
0 & 0 & S_{66}
\end{array}\right]\left\{\begin{array}{l}
\sigma_{1} \\
\sigma_{2} \\
\tau_{12}
\end{array}\right\} \text { or }\left\{\varepsilon^{1}\right\}=[S]\left\{\sigma^{1}\right\}
$$

where $[S]$ is the axis flexibility matrix. The equation (10) can also be given as,

$$
\left\{\begin{array}{l}
\varepsilon_{1} \\
\varepsilon_{2} \\
\gamma_{12}
\end{array}\right\}=\left[\begin{array}{ccc}
\frac{1}{E_{1}} & -\frac{v_{12}}{E_{2}} & 0 \\
-\frac{v_{21}}{E_{1}} & \frac{1}{E_{2}} & 0 \\
0 & 0 & \frac{1}{G_{12}}
\end{array}\right]\left\{\begin{array}{l}
\sigma_{1} \\
\sigma_{2} \\
\tau_{12}
\end{array}\right\}
$$

The inverse of the equation (10) is available,

$$
\left\{\begin{array}{l}
\sigma_{1} \\
\sigma_{2} \\
\tau_{12}
\end{array}\right\}=\left[\begin{array}{ccc}
Q_{11} & Q_{12} & 0 \\
Q_{21} & Q_{22} & 0 \\
0 & 0 & Q_{66}
\end{array}\right]\left\{\begin{array}{l}
\varepsilon_{1} \\
\varepsilon_{2} \\
\gamma_{12}
\end{array}\right\} \text { or }\left\{\sigma^{1}\right\}=[Q]\left\{\varepsilon^{1}\right\}
$$

where $[Q]$ is the converted axis stiffness matrix at the plane stress state. $Q_{i j}$ in equation (12) and $C_{i j}$ in equation (2) have the following relations,

$$
\begin{array}{llll}
Q_{11} & =C_{11} & -C_{13} C_{13} & =C_{33} \\
12 & 12 & 13 & 23 \\
22 & 22 & 23 & 23 \\
2 & &
\end{array}
$$

The values of the flexibility and stiffness in equation (11) and equation (13) can be obtained through micromechanics calculations or experiments. 


\subsection{Off-axis flexibility and stiffness of monolayer under plane stress}

The off-axis flexibility and stiffness are often used in the mechanical analysis on the unidirectional fiber monolayer. As displayed in Fig. 4, axes 1 and 2 are along the principal axes of material, $x$ and $y$ are off-axis, and the anticlockwise angle from $x$-axis to the 1 -axis is positive. Thus, we obtain,

$$
\begin{array}{r}
\left\{\begin{array}{l}
\sigma_{1} \\
\sigma_{2} \\
\tau_{12}
\end{array}\right\}^{x}=\left[\begin{array}{ccc}
\cos ^{2} \theta & \sin ^{2} \theta & -2 \sin \theta \cos \theta \\
\sin ^{2} \theta & \cos ^{2} \theta & 2 \sin \theta \cos \theta \\
\sin \theta \cos \theta & -\sin \theta \cos \theta & \cos ^{2} \theta-\sin ^{2} \theta
\end{array}\right]\left\{\begin{array}{l}
\sigma_{1} \\
\sigma_{2} \\
\tau_{12}
\end{array}\right\}^{1} \\
\text { or }\left\{\sigma^{x}\right\}=[T]\left\{\sigma^{1}\right\}
\end{array}
$$

and

$$
\begin{array}{r}
\left\{\begin{array}{l}
\sigma_{1} \\
\sigma_{2} \\
\tau_{12}
\end{array}\right\}^{1}=\left[\begin{array}{ccc}
\cos ^{2} \theta & \sin ^{2} \theta & 2 \sin \theta \cos \theta \\
\sin ^{2} \theta & \cos ^{2} \theta & -2 \sin \theta \cos \theta \\
-\sin \theta \cos \theta & \sin \theta \cos \theta & \cos ^{2} \theta-\sin ^{2} \theta
\end{array}\right]\left\{\begin{array}{l}
\sigma_{1} \\
\sigma_{2} \\
\tau_{12}
\end{array}\right\}^{x} \\
\text { or }\left\{\sigma^{1}\right\}=[T]^{-1}\left\{\sigma^{x}\right\}
\end{array}
$$

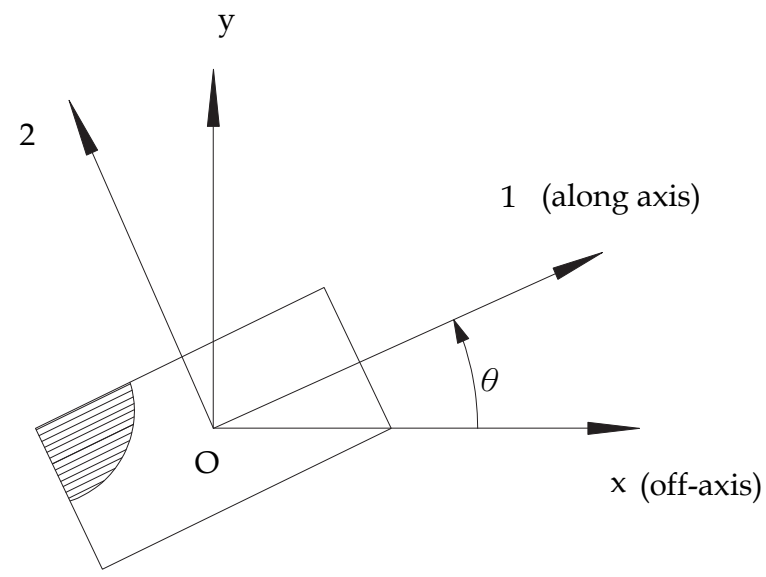

Fig. 4. Sketch of coordinate transformation between the axis and off-axis of monolayer with unidirectional fibers

If, we set,

$$
[R]=\left[\begin{array}{lll}
1 & & \\
& 1 & \\
& & 2
\end{array}\right]
$$




$$
[F]=[R][T][R]^{-1}\left[\begin{array}{ccc}
\cos ^{2} \theta & \sin ^{2} \theta & -\sin \theta \cos \theta \\
\sin ^{2} \theta & \cos ^{2} \theta & \sin \theta \cos \theta \\
2 \sin \theta \cos \theta & -2 \sin \theta \cos \theta & \cos ^{2} \theta-\sin ^{2} \theta
\end{array}\right]
$$

the follow can be obtained,

$$
\left\{\varepsilon^{x}\right\}=[F]\left\{\varepsilon^{1}\right\}
$$

It should be known from the equation (15) and (17) that,

$$
[F]^{T}=[T]^{-1}
$$

So that,

$$
[F]^{-1}=[T]^{T}
$$

Thus, the inverse function of the equation (18) is,

$$
\left\{\varepsilon^{1}\right\}=[F]^{-1}\left\{\varepsilon^{x}\right\}=[T]^{T}\left\{\varepsilon^{x}\right\}
$$

With these relations, the follows can be given,

$$
\begin{gathered}
\left\{\sigma^{x}\right\}=[T]\left\{\sigma^{1}\right\}=[T][Q]\left\{\varepsilon^{1}\right\}=[T][Q][T]^{T}\left\{\varepsilon^{x}\right\} \\
\text { or }\left\{\sigma^{x}\right\}=[\bar{Q}]\left\{\varepsilon^{x}\right\}
\end{gathered}
$$

and

$$
\begin{array}{r}
\left\{\varepsilon^{x}\right\}=[F]\left\{\varepsilon^{1}\right\}=[F][S]\left\{\sigma^{1}\right\}=[F][S][T]^{-1}\left\{\sigma^{x}\right\}=[F][S][F]^{T}\left\{\sigma^{x}\right\} \\
\text { or }\left\{\varepsilon^{x}\right\}=[\bar{S}]\left\{\sigma^{x}\right\}
\end{array}
$$

The equation (22) and (23) are the constitutive equations in the Oxy coordinate system, where the off-axis stiffness $[\bar{S}]$ and the off-axis flexibility $[\bar{Q}]$ are given as follows,

$$
\begin{gathered}
{[\bar{Q}]=\left[\begin{array}{lll}
\bar{Q}_{11} & \bar{Q}_{12} & \bar{Q}_{16} \\
\bar{Q}_{21} & \bar{Q}_{22} & \bar{Q}_{26} \\
\bar{Q}_{61} & \bar{Q}_{62} & \bar{Q}_{66}
\end{array}\right]=[T][Q][T]^{T}} \\
{[\bar{S}]=\left[\begin{array}{lll}
\bar{S}_{11} & \bar{S}_{12} & \bar{S}_{16} \\
\bar{S}_{21} & \bar{S}_{22} & \bar{S}_{26} \\
\bar{S}_{61} & \bar{S}_{62} & \bar{S}_{66}
\end{array}\right]==[F][S][F]^{T}}
\end{gathered}
$$

In the above two equations, 


$$
\bar{Q}_{i j}=\bar{Q}_{j i}, \quad \bar{S}_{i j}=\bar{S}_{j i}
$$

The off-axis stiffness is,

$$
\begin{aligned}
& \bar{Q}_{11}=Q_{22} \cos ^{4} \theta+2\left(Q_{12}+2 Q_{66}\right) \sin ^{2} \theta \cos ^{2} \theta+Q_{22} \sin ^{4} \theta \\
& \bar{Q}_{12}=Q_{66}+\left(Q_{11}+Q_{22}-2 Q_{12}-4 Q_{66}\right) \sin ^{2} \theta \cos ^{2} \theta \\
& \bar{Q}_{16}= \pm\left(Q_{11}-Q_{12}-2 Q_{66}\right) \sin \theta \cos ^{3} \theta \mp\left(Q_{22}-Q_{12}-2 Q_{66}\right) \sin ^{3} \theta \cos \theta
\end{aligned}
$$

And the off-axis flexibility,

$$
\begin{aligned}
& \bar{S}_{11}=S_{11} \cos ^{4} \theta+\left(2 S_{12}+S_{66}\right) \sin ^{2} \theta \cos ^{2} \theta+S_{22} \sin ^{4} \theta \\
& \bar{S}_{12}=S_{12}+\left(S_{11}+S_{22}-2 S_{12}-S_{66}\right) \sin ^{2} \theta \cos ^{2} \theta \\
& \bar{S}_{66}=S_{66}+4\left(S_{11}+S_{22}-2 S_{12}-S_{66}\right) \sin ^{2} \theta \cos ^{2} \theta \\
& \bar{S}_{16}= \pm \underset{22}{\left(2 S_{11}-2 S_{12}-S_{66}\right) \sin \theta \cos ^{3} \theta \mp\left(2 S_{22}-2 S_{12}-S_{66}\right) \sin ^{3} \theta \cos \theta}
\end{aligned}
$$

\subsection{Constitutive equations in classical laminated plate theory}

The so-called classical laminated plate theory or the classical laminate theory, refers to the use of the straight normal hypothesis in elastic shell theory, neglects a number of secondary factors, and has been an acknowledged laminated plate theory. In the classical laminated plate theory, the transverse shear strain $\gamma_{23}$ and $\gamma_{31}$, and the normal direction strain $\varepsilon_{3}$ are supposed to be zero.

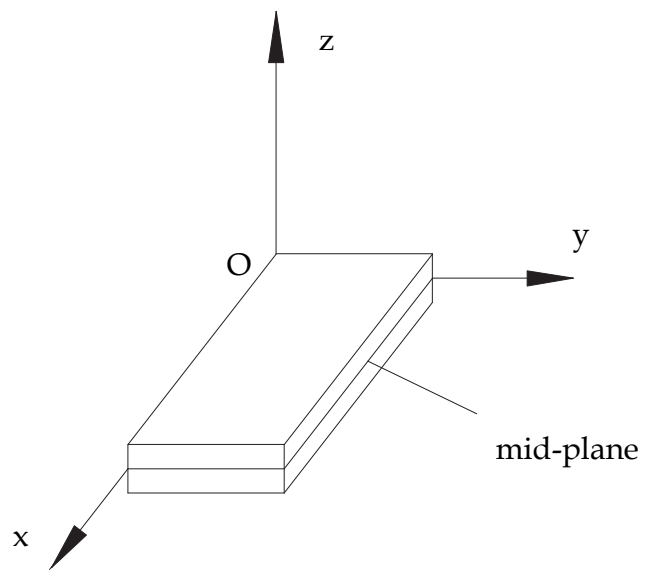

Fig. 5. Sketch of laminated thin plate and the Cartesian coordinates

As a result of straight normal assumption, the deformation of laminated plate can be described with the mid-plane deformation. If the mid-plane strain is $\varepsilon^{0}$ and thickness of 
each layer in the laminated plate is the same, the mid-plane stress of an n-layers laminated plate is

$$
\left\{\sigma^{0}\right\}=\frac{1}{n} \sum_{k=1}^{n}\left\{\sigma^{(k)}\right\}=\frac{1}{n} \sum_{k=1}^{n}\left([\bar{Q}]^{(k)}\left\{\varepsilon^{0}\right\}\right)=\left[\frac{1}{n} \sum_{k=1}^{n}\left([\bar{Q}]^{(k)}\right)\right] \cdot\left\{\varepsilon^{0}\right\}
$$

where $\sigma^{0}$ is the mid-plane stress of the laminated plate, $\sigma^{(k)}$ is the stress of the $k$ th layer, $[\bar{Q}]^{(k)}$ is the converted stiffness of the $k$ th layer.

\section{Equivalent model of graphite sheet}

\subsection{The basic idea of the equivalent model}

All the $\mathrm{C}$ atoms in the graphite sheet are connected with the o bonds and the bonds form a hexagonal structure (Fig.6). Fig. 7 is a schematic diagram of the graphite sheet, thick solid lines in which represent the C-C bond in graphite. If each $\mathrm{C}-\mathrm{C}$ bond is longer (shown in thin lines), that will form a network structure, as shown in Fig. 8.

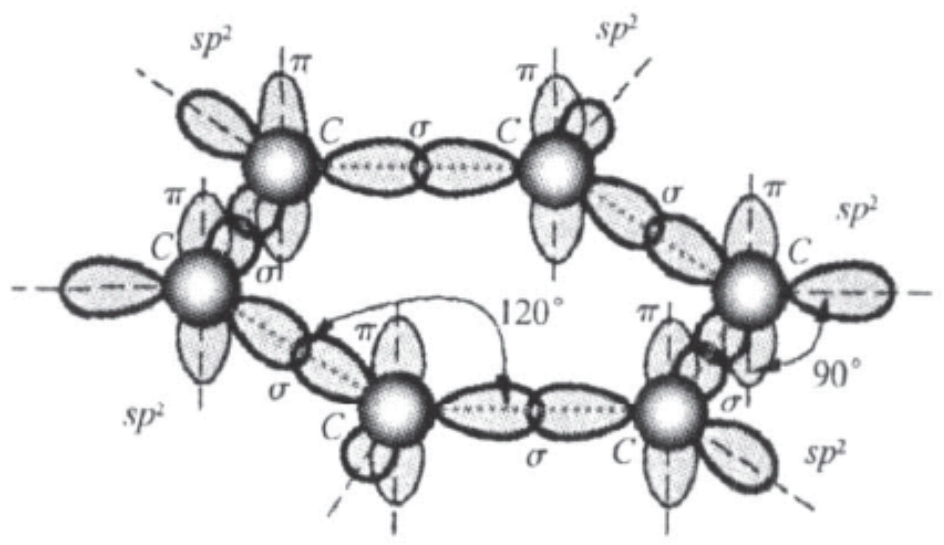

Fig. 6. The bonding relationship in C-C covalent bonds

As can be seen from Fig. 8, the network structure is formed by the three groups of parallel fibers and they are into 60 degree angles with each other. If we consider each group of fiber as a composite monolayer, the mechanical properties of the entire network structure can be obtained with the laminated plate theory. Comparing Fig. 7 and Fig. 8, we find that the network structure in Fig. 8 can also be formed if the three graphite sheets in Fig. 7 are staggered and stacked one on top of the other.

In summary, here we put forward a new original equivalent model used to study the mechanical properties of graphite sheet. The analysis steps are, treat the network structure shown in Fig. 8 as a laminated composite plate with three layers orthotropic monolayer of unidirectional fiber (each fiber in the monolayer is just the covalent $\mathrm{C}-\mathrm{C}$ bond in series), the mechanical properties of fiber can be deduced from the physical parameters of graphite, and the $1 / 3$ of the converted stiffness of the network structure can be considered as the converted stiffness of the graphite sheet at the plane stress state. 


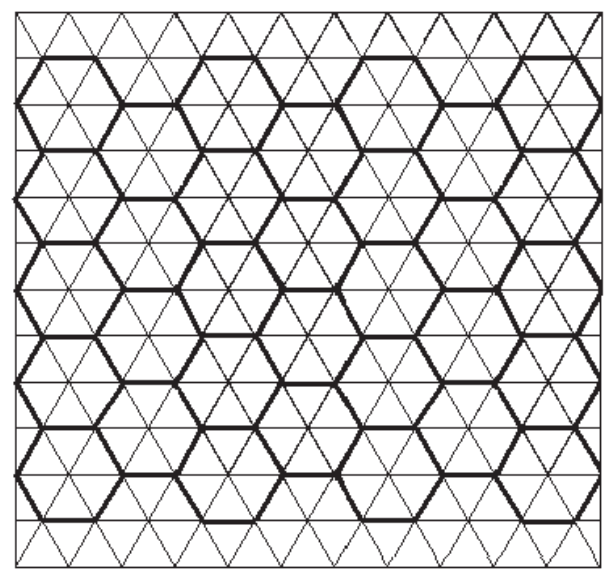

Fig. 7. The atomic structure of a graphite sheet

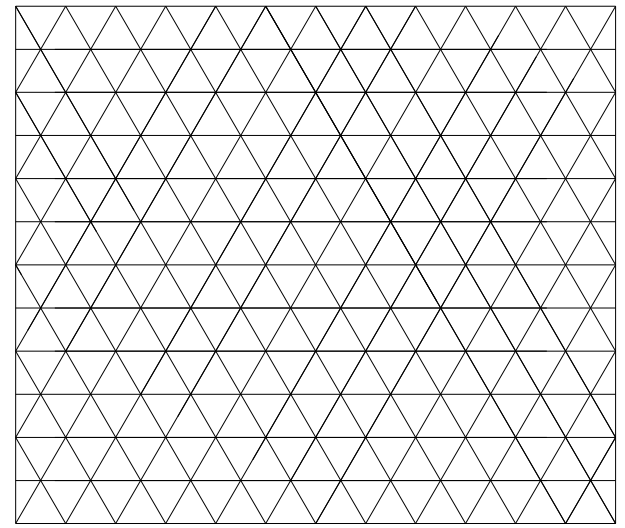

Fig. 8. Effective network structure of laminated graphite sheets

\subsection{Mechanical properties of graphite sheet at plane stress state}

The hexagonal plane composed of the $\sigma$ bonds is defined as the $\sigma$-plane, and the energy of interactions between any $\mathrm{C}$ atoms in the o-plane are considered to be functions of the position of the $\mathrm{C}$ atoms. With all the weak interactions (e.g. the electronic potential, the van der Waals interactions) neglected, the total potential energy of the graphite sheet can be expressed as.

$$
U_{\text {graphite }}=U_{r}+U_{\theta}
$$

where $U_{r}$ is the axial stretching energy of $\mathrm{C}-\mathrm{C}$ bond, $U_{\theta}$ is the $\mathrm{C}-\mathrm{C}-\mathrm{C}$ bond angle potential. Establish a local coordinate system in the $\sigma$-plane with the $\mathrm{C}-\mathrm{C}$ bond direction as the $\mathrm{x}^{\prime}$ axis (Fig. 9), we can obtain the whole potential, 


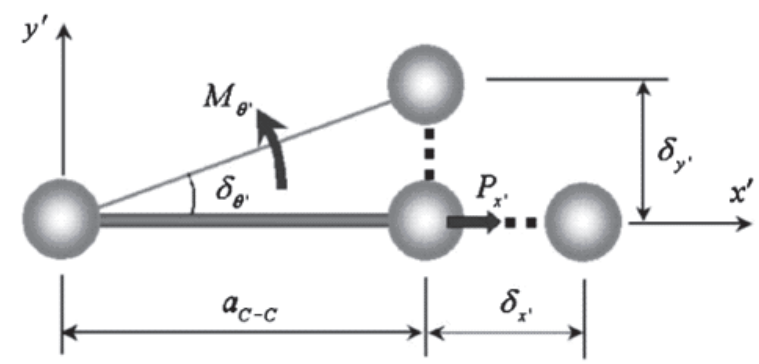

Fig. 9. Local element coordinates of C-C bond in graphite

$$
U_{C-C}=U_{x^{\prime}}+U_{\theta^{\prime}}
$$

where $U_{x^{\prime}}$ is the axial stretching energy of C-C bond in the local coordinate system and $U_{\theta^{\prime}}$ the angle bending energy. According to the basic principles of molecular mechanics, the two bond energy can be written as:

$$
\begin{aligned}
& U_{x^{\prime}}=\frac{1}{2} k_{x^{\prime}} \cdot \delta_{x^{\prime}}^{2} \\
& U_{\theta^{\prime}}=\frac{1}{2} k_{\theta^{\prime}} \cdot \delta_{\theta^{\prime}}^{2}
\end{aligned}
$$

where $\delta_{x^{\prime}}$ and $\delta_{\theta^{\prime}}$ are the displacement, $k_{x^{\prime}}$ and $k_{\theta^{\prime}}$ are the MD force field constants.

\subsection{Elastic constants of monolayer in the equivalent model}

If we consider the $\mathrm{C}-\mathrm{C}$ bond as a single elastic fiber in the equivalent model, and the 1 axis for the fiber is defined as the axial direction of the $\mathrm{C}-\mathrm{C}$ bond, we have,

$$
\begin{gathered}
K_{1}=k_{x^{\prime}} \\
E_{1}=\frac{K_{1} \cdot a_{C-C}}{A_{C-C}}=\frac{k_{x} \cdot a_{C-C}}{A_{C-C}}
\end{gathered}
$$

where $K_{1}$ is the elastic stiffness factor of the fiber at the 1 direction, $a_{C-C}$ and $A_{C-C}$ are the $\mathrm{C}-\mathrm{C}$ bond length and the cross-sectional area of the equivalent fiber, $E_{1}$ is the elastic modulus of the fiber at the 1 direction.

The 2 axis for the fiber is defined as the vertical direction of the $\mathrm{C}-\mathrm{C}$ bond in the o-plane. The fiber interactions at the 2 direction are mainly reflected through the $\mathrm{C}-\mathrm{C}-\mathrm{C}$ angle bending potential (Fig. 10).

Set $K_{2}$ to be the elastic stiffness factor of the fiber at the 2 direction, we obtain,

$$
2 \cdot\left[2 \cdot \frac{1}{2} k_{\theta} \cdot \delta_{\theta}^{2}+\frac{1}{2} k_{\theta^{\prime}} \cdot\left(2 \delta_{\theta}\right)^{2}\right]=\frac{1}{2} K_{2} \cdot \delta_{2}^{2}=\frac{1}{2} K_{2} \cdot\left(2 \cdot a_{C-C} \cdot \delta_{\theta} \cdot \sin \frac{\pi}{6}\right)^{2}
$$




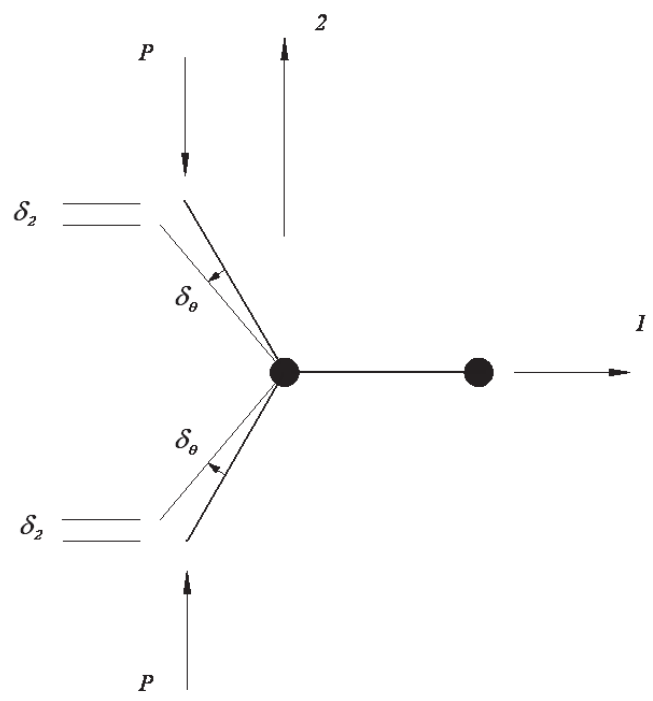

Fig. 10. Loading on the direction 2 of fiber in the equivalent model of graphite

$$
\begin{gathered}
K_{2}=\frac{12 \cdot k_{\theta^{\prime}}}{a_{C-C}^{2}} \\
E_{2}=\frac{1}{2} \frac{K_{2} \cdot \sqrt{3} \cdot a_{C-C}}{t \cdot\left(a_{C-C}+a_{C-C} \cdot \sin \frac{\pi}{6}\right)}=\frac{\sqrt{3} K_{2}}{3 t}=\frac{4 \sqrt{3} k_{\theta^{\prime}}}{3 t \cdot a_{C-C}^{2}}
\end{gathered}
$$

where $\delta_{\theta}$ and $\delta_{2}$ are the C-C-C angle change and the displacement at the 2 direction when the model is loaded at the 2 direction, $t$ is the effective thickness of the equivalent fiber layer, $E_{2}$ is the elastic modulus of the fiber at the 2 direction.

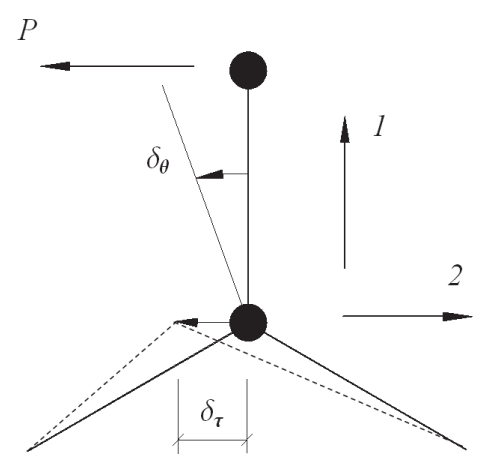

Fig. 11. Shearing deformation of the fiber in the equivalent model of graphite 
When the fiber layer in the equivalent model is subjected to a shearing force $P$ (Fig. 11), there will be a horizontal displacement $\delta_{\tau}$ for the equivalent fiber element and an angle deflection $\delta_{\theta}$. As to the displacement of the bottom atom in of the figure, it can be obtained,

$$
P=2 \cdot\left[K_{1} \cdot\left(\delta_{\tau} \cdot \cos \frac{\pi}{6}\right)\right]=\sqrt{3} K_{1} \delta_{\tau}
$$

The strain energy induced by the angle deflection $\delta_{\theta}$ of a fiber is,

$$
2 \cdot\left(2 \cdot \frac{1}{2} k_{\theta} \cdot \delta_{\theta}^{2}\right)=\frac{1}{2} P \cdot a_{C-C} \cdot \delta_{\theta}
$$

Substitute equation (38) into (39), we obtain,

$$
a_{C-C} \cdot \delta_{\theta}=\frac{\sqrt{3} K_{1} \delta_{\tau} \cdot a_{C-C}^{2}}{4 k_{\theta^{\prime}}}=\frac{\sqrt{3} K_{1}}{K_{2} / 3} \delta_{\tau}
$$

Therefore, the shearing deformation of the fiber element in the equivalent model is,

$$
\bar{\delta}_{\tau}=\delta_{\tau}+a_{C-C} \cdot \delta_{\theta}=\left(1+\frac{3 \sqrt{3} K_{1}}{K_{2}}\right) \delta_{\tau}
$$

According to the definition of the elastic shear modulus:

$$
G_{12}=\frac{\tau_{12}}{\gamma_{12}}
$$

for the fiber element in the equivalent model, there is:

$$
G_{12}=\frac{P /\left(t \cdot 2 \cdot a_{C-C} \cdot \cos \frac{\pi}{6}\right)}{\frac{1}{2} \bar{\delta}_{\tau} / a_{C-C}}=\frac{2 P}{\sqrt{3} \bar{\delta}_{\tau} \cdot t}
$$

We substitute equation (38) and (41) into (43), and get

$$
G_{12}=\frac{2 K_{1} \delta_{\tau}}{t \bar{\delta}_{\tau}}=\frac{2 K_{1}}{t\left(1+\frac{3 \sqrt{3} K_{1}}{K_{2}}\right)}
$$

\subsection{Test for the mechanical constants of the monolayer in the equivalent model}

From equation (34), (37) and (44), we obtain the mechanical constants of the monolayer in the equivalent model $E_{1}, E_{2}$ and $G_{12}$, of which $E_{1}$ and $E_{2}$ are independent quantities, $G_{12}$ is a function of $E_{1}$ and $E_{2}$. Both $E_{1}$ and $E_{2}$ are related to MD parameters of the C-C bond energy There are several empirical potentials and relevant parameters for $\mathrm{C}-\mathrm{C}$ bond energy. In this section, the following potential energy function and parameters are used to verify the equivalent model provided in this chapter. The Morse potential is employed for the bond 
stretching action and harmonic potential for the angle bending. The short-range potential caused by the deformation of C-C bond is described as bellow,

$$
\begin{gathered}
U_{r}=K_{r}\left(1-e^{-\beta\left(r_{i j}-r_{0}\right)}\right)^{2} \\
U_{\theta}=\frac{1}{2} K_{\theta}\left(\theta_{i j k}-\theta_{0}\right)^{2}
\end{gathered}
$$

where $U_{r}$ and $U_{\theta}$ are the potentials of bond stretching and angle bending, $K_{r}$ and $K_{r}$ are the corresponding force constants. $r_{i j}$ represents the distance between any couple of bonded atoms, $\theta_{i j k}$ represents all the possible angles of bending, $r_{0}$ and $\theta_{0}$ are the corresponding reference geometry parameters of grapheme. $\beta$ defines the steepness of the Morse well. The values of all these parameters are listed in Table 1.

$$
\text { Bond } \quad K_{r}=478.9 \mathrm{KJ} / \mathrm{mol}, \beta=21.867 \mathrm{~nm}^{-1}, r_{0}=0.142 \mathrm{~nm}
$$

$$
\text { Angle } \quad K_{\theta}=418.4 \mathrm{KJ} / \mathrm{mol}, \theta_{0}=120.00^{\circ}
$$

Table 1. Parameters for C-C bond in MD

Comparing equation (32) and (45), using the data in Table 1, we can obtain:

$$
K_{1}=k_{x^{\prime}}=\beta^{2} \cdot 2 K_{r}=760.4 \mathrm{nN} / \mathrm{nm}
$$

We substitute data in Table 1 into equation (36), and get

$$
K_{2}=\frac{12 \cdot k_{\theta^{\prime}}}{a_{C-C}^{2}}=413.48 \mathrm{nN} / \mathrm{nm}
$$

There are other scholars working on the C-C stretching force constants through experiments or theoretical calculations, who reported the values along the $\mathrm{C}-\mathrm{C}$ bond like $729 \mathrm{nN} / \mathrm{nm}, 880 \mathrm{nN} / \mathrm{nm}, 708 \mathrm{nN} / \mathrm{nm}$ and so on. They also obtained the constants at the direction perpendicular to the C-C bond, $432 \mathrm{nN} / \mathrm{nm}$ and $398 \mathrm{nN} / \mathrm{nm}$ (Yang \& Zeng, 2006). Noting equation (47) and (48), we can see that the data obtained here based on the current equivalent model are in good agreement with the results from other researchers.

\section{Elastic properties of graphite sheet}

\subsection{Flexibility of monolayer in the equivalent model}

Graphite sheet can be considered as the network structure formed by the three groups of parallel fibers which are into 60 degree angles with each other. Based on the result of the last section, we can get the axial flexibility of the fiber monolayer, 


$$
[S]^{(1)}=\left[\begin{array}{ccc}
\frac{1}{E_{1}} & -\frac{v_{12}}{E_{2}} & 0 \\
-\frac{v_{21}}{E_{1}} & \frac{1}{E_{2}} & 0 \\
0 & 0 & \frac{1}{G_{12}}
\end{array}\right]
$$

where it has $\frac{v_{12}}{E_{2}}=\frac{v_{21}}{E_{1}} . E_{1}, E_{2}$ and $G_{12}$ can be calculated according to the equation (34), (37) and (44). If we consider $v_{21}$ to be 0.3 with reference to the parameters of general materials, assume the fiber thickness of $0.34 \mathrm{~nm}$, and apply the data in Table 1 , the follows can be calculated,

$$
\begin{aligned}
& E_{1}=1.190 \mathrm{TPa}, E_{2}=0.702 \mathrm{TPa}, G_{12}=0.424 \mathrm{TPa} \\
& \frac{v_{12}}{E_{2}}=\frac{v_{21}}{E_{1}}=0.252
\end{aligned}
$$

Substituting the values in equation (50) into equation (49), we get the axis flexibility of the monolayer,

$$
[S]^{(1)}=\left[\begin{array}{ccc}
0.8403 & -0.252 & 0 \\
-0.252 & 1.4245 & 0 \\
0 & 0 & 2.360
\end{array}\right]
$$

where the unit of the values is $10^{-3} \mathrm{~nm}^{2} / \mathrm{nN}$.

Substituting $\theta=\pi / 3$ and the values in equation (51) into equation (28), we get the off-axis flexibility of the $60^{\circ}$ and $-60^{\circ}$ monolayers,

$$
\begin{aligned}
& {[\bar{S}]^{\left(60^{\circ}\right)}=\left[\begin{array}{ccc}
1.2038 & -0.1743 & -0.3444 \\
-0.1743 & 0.9097 & -0.1650 \\
-0.3444 & -0.1650 & 2.6693
\end{array}\right]} \\
& {[\bar{S}]^{\left(-60^{\circ}\right)}=\left[\begin{array}{ccc}
1.2038 & -0.1743 & 0.3444 \\
-0.1743 & 0.9097 & 0.1650 \\
0.3444 & 0.1650 & 2.6693
\end{array}\right]}
\end{aligned}
$$

The unit of the values is also $10^{-3} \mathrm{~nm}^{2} / \mathrm{nN}$ in the last two equations.

\subsection{Elastic properties of the equivalent model of graphite}

The graphite sheet is a whole layer structure with no delamination and the strain did not change in the thickness. Thus we can use formula (29) to calculate the stiffness,

$$
[\bar{Q}]=\frac{1}{3} \sum_{k=1}^{3}[\bar{Q}]^{(k)}
$$


where $[\bar{Q}]$ is the converted stiffness of the graphite sheet, $[\bar{Q}]^{(k)}$ is the stiffness of the $k$ th layer in the equivalent model. The flexibility of the graphite sheet is,

$$
[\bar{S}]=[\bar{Q}]^{-1}=3 / \sum_{k=1}^{3}[\bar{Q}]^{(k)}
$$

Substituting the values in equation (51) (53) into (55), we can obtain,

$$
[\bar{S}]=\frac{3}{\left\{[S]^{(1)}\right\}^{-1}+\left\{[\bar{S}]^{\left(60^{\circ}\right)}\right\}^{-1}+\left\{[\bar{S}]^{\left(-60^{\circ}\right)}\right\}^{-1}}=\left\{\begin{array}{ccc}
1.0251 & -0.2063 & 0 \\
-0.2063 & 1.0251 & 0 \\
0 & 0 & 2.4628
\end{array}\right\}
$$

where the unit of the values is $10^{-3} \mathrm{~nm}^{2} / \mathrm{nN}$.

A series of well acknowledged experiment values (Yang \& Zeng, 2006) of elastic constants of perfect graphite are listed in Table 2. Data obtained in current work are in good agreement with those results, which justifies the present equivalent model. It should be noticed that there is an obvious error of the $S_{12}$. However, $S_{12}$ has little effect on the mechanical properties of the graphite sheet, the error on it will not influence the reasonable application of the current equivalent model in the mechanics analysis of graphite.

\begin{tabular}{cccc}
\hline Items & $S_{11} / 10^{-3} \mathrm{~nm}^{2} / \mathrm{nN}$ & $S_{12} / 10^{-3} \mathrm{~nm}^{2} / \mathrm{nN}$ & $S_{66} / 10^{-3} \mathrm{~nm}^{2} / \mathrm{nN}$ \\
\hline Experiment & 0.98 & -0.16 & 2.28 \\
\hline Current & 1.0251 & -0.2063 & 2.4628 \\
\hline Error & $4.60 \%$ & $28.94 \%$ & $8.02 \%$ \\
\hline
\end{tabular}

Table 2. Data obtained in current work and elastic constants of perfect graphite crystals from other experiment

Both the result in equation (56) and the data listed in Table 2 indicate that the graphite is inplane isotropic at plane stress state. And according to the classical theory of composite mechanics, it is known that the $\pi / m$ laminated plates with $m \geq 3$ are in-plane isotropic. We can see that the graphite sheets being in-plane isotropic is mainly due to their special structure of C-C-C angles.

According to the points made above, we try to explain why the elastic properties of CNTs are anisotropic to some extent. One of the possible reasons is that the curling at different curvature from graphene to CNTs makes the C-C-C angles change (e.g. the angles in an armchair CNT with $1 \mathrm{~nm}$ diameter are not fixed $120^{\circ}$, but about 118 ), for which the quasiisotropy of graphite sheet is disrupted and the orthotropy introduced. Having an general realization of the CNTs, one should find that the change of the C-C-C angle is obviously related to the change of $\mathrm{CNT}$ radius, especially when it is a small tube in diameter. Thus, the 
orthotropy of CNTs is diameter related, which is in good agreement with the other research results referring to the mechanical properties of CNTs changing due to their various radial size. The changes of C-C-C angles are also somehow related to the chirality of CNTs. However, the extent of the changes of chiral angles is about $0^{\circ} \sim 30^{\circ}$, and the change of C-C$\mathrm{C}$ induced by the difference of chiral angles is little when to curl the graphite sheet at the same curvature. It agrees with that many studies reporting that the difference of elastic properties among various chiral CNTs decreases with the increase in the diameter of CNTs.

\subsection{Elastic properties of the equivalent model with various $\mathrm{C}-\mathrm{C}-\mathrm{C}$ bond angles}

The C-C-C bond angles will change from the constant $120^{\circ}$ to smaller values when the graphite sheet curling to CNTs. In the same way, the angles between the fibers in the equivalent model will change to less than $\pi / 3$ and the effect of the change on the elastic properties of the model will be investigated in this section.

Substituting equation (51) and the angle $\theta^{\prime}$ between the fibers (responding to the changed C-C-C angles) into equation (28), we can obtain the off-axis flexibility of $\theta^{\prime}$ and $-\theta^{\prime}$ monolayers, $[\bar{S}]^{\left(\theta^{\prime}\right)}$ and $[\bar{S}]^{\left(-\theta^{\prime}\right)}$. Substitute those two into equation (55) to get the approximate converted flexibility of the equivalent model (responding to the changed C-CC angles):

$$
\left[\bar{S}_{\bar{\theta}^{\prime}}\right]=\frac{3}{\left\{[S]^{(1)}\right\}^{-1}+\left\{[\bar{S}]^{\left(\theta^{\prime}\right)}\right\}^{-1}+\left\{[\bar{S}]^{\left(-\theta^{\prime}\right)}\right\}^{-1}}
$$

The elastic modulus of the equivalent model responding to the graphite with changed C-C$\mathrm{C}$ angles, at the direction of $0^{\circ}$ fiber or at the vertical direction, can be obtained from equation (57). The variation of the modulus with the changes of the C-C-C angles are displayed in Fig. 12 and Fig. 13, and also displayed the variation of $G_{12}, v_{12}$ and $v_{21}$ in Fig.14 Fig.16.

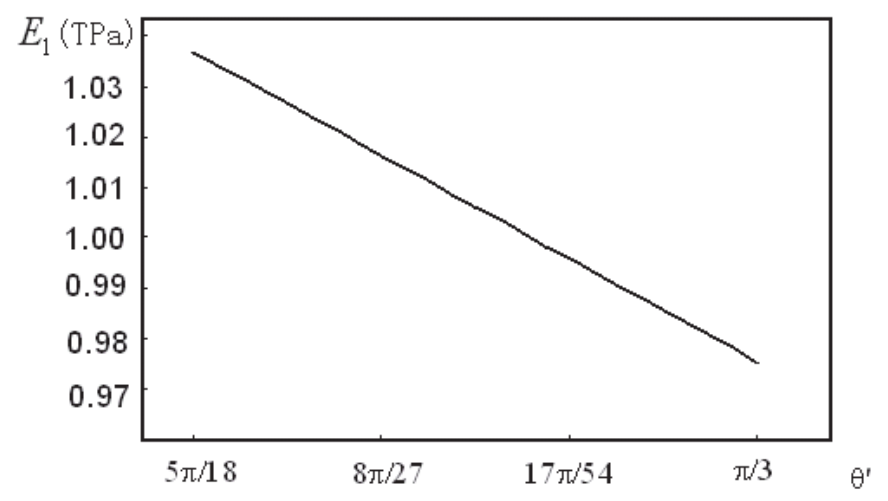

Fig. 12. Elastic modulus of the equivalent model of graphene along the 0 o fiber 


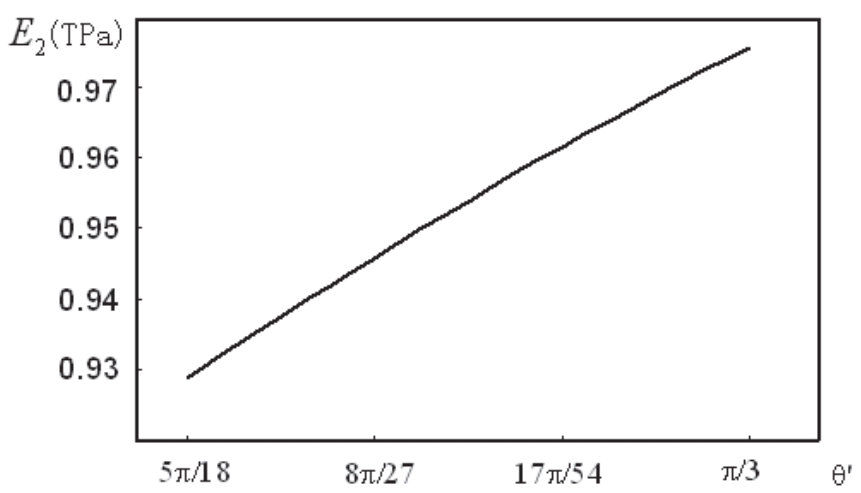

Fig. 13. Elastic modulus of the equivalent model of graphene at perpendicular direction to the $0^{\circ}$ fiber

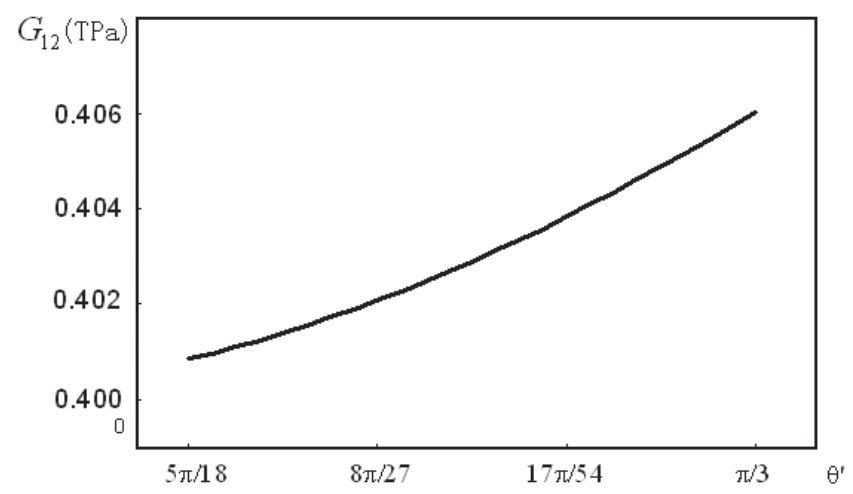

Fig. 14. $\mathrm{G}_{12}$ of the equivalent model of graphene

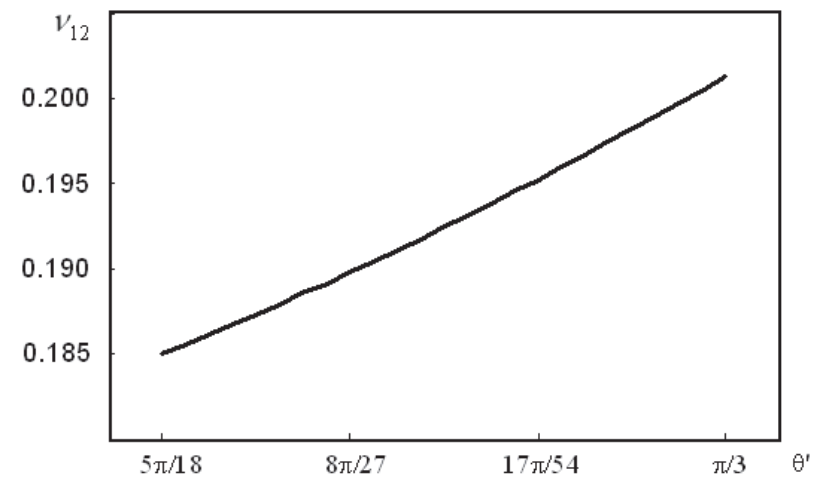

Fig. 15. $v_{12}$ of the equivalent model of graphene 


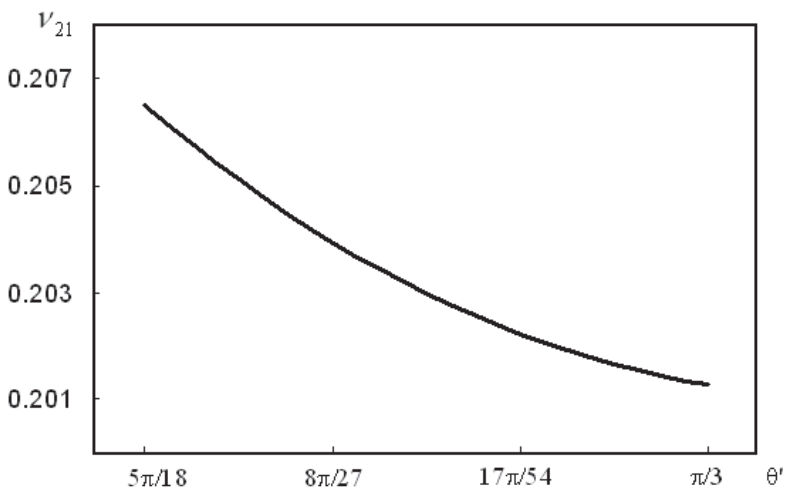

Fig. 16. $v_{21}$ of the equivalent model of graphene

We can see from Fig. 12 and Fig. 13 that the elastic modulus along the 0 o fiber of the equivalent model of graphene almost increases linearly, and the elastic modulus at the vertical direction decreases, with the decrease in the C-C-C angle. And Fig. $14 \sim$ Fig. 16 show that the $\mathrm{G}_{12}, v_{12}$ and $v_{21}$ of the equivalent model also changes a lot with the changes of the C-C-C angle.

\section{Scale effect of elastic properties of CNTs}

\subsection{Equivalent model of single-walled carbon nanotubes (SWCNTs)}

$\mathrm{CNTs}$ can be considered as curling graphite sheet. The C-C-C bond angles will change from the constant $120^{\circ}$ to smaller values when the graphite sheet curling to CNTs and the change of the C-C-C angle is related to the radius of the formed CNTs. According to that, we consider CNTs same as graphene with changed C-C-C angles and the elastic properties of $\mathrm{CNTs}$ are consistent with those of graphene with changed $\mathrm{C}-\mathrm{C}-\mathrm{C}$ angles.

\subsubsection{Zigzag SWCNTs}

Taking the zigzag SWCNTs as an example, we study the effect of the changing in diameter of SWCNTs on the value of C-C-C angle. The SWCNT structure and the geometric diagram are shown in Fig. 17, with which we can obtain,

$$
\begin{aligned}
& \sin \angle D B F=\frac{D F}{D B} \\
& \sin \angle C E F=\frac{C F}{C E}
\end{aligned}
$$

With $D F=C F$, the last equation becomes,

$$
\sin \angle D B F=\frac{C E}{D B} \cdot \sin \angle C E F \approx \frac{\sqrt{3}}{2} \sin \angle C E F
$$


where the $\angle B D E$ does not change a lot when the graphene curls to the SWCNT so that we make $\frac{C E}{D B}=\frac{D E}{D B} \approx \frac{\sqrt{3}}{2}$.

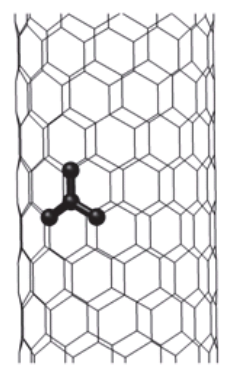

(a)

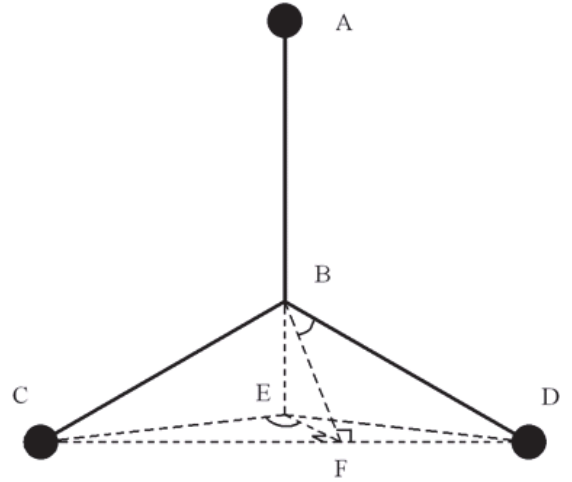

(b)

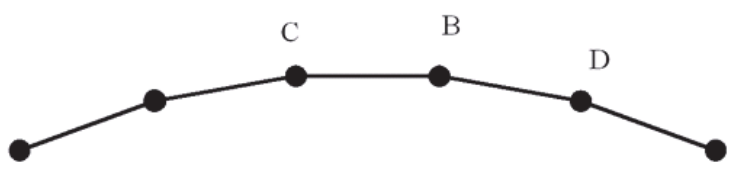

(c)

Fig. 17. C-C-C angles in the zigzag SWCNTs

For an $(\mathrm{m}, 0)$ zigzag SWCNT, it has,

$$
\angle C E F=\frac{(m-1) \pi}{2 m}
$$

The diameter of the tube is,

$$
d_{c n t}=0.0783 \cdot m \quad \mathrm{~nm}
$$

Substituting equation (61) and (60) into (59), we get,

$$
\sin \angle D B F=\frac{\sqrt{3}}{2} \sin \left[\frac{\pi}{2}\left(1-\frac{0.0783}{d_{c n t}}\right)\right]
$$

Thus, with the diameter of the zigzag SWCNT provided, setting the AB direction in Fig. 17 as the direction of the 0 o fiber, we can calculate the C-C-C angles in zigzag SWCNTs as,

$$
\theta^{\prime}=\operatorname{Arcsin}\left\{\frac{\sqrt{3}}{2} \cdot \sin \left[\frac{\pi}{2}\left(1-\frac{0.0783}{d_{c n t}}\right)\right]\right\}
$$




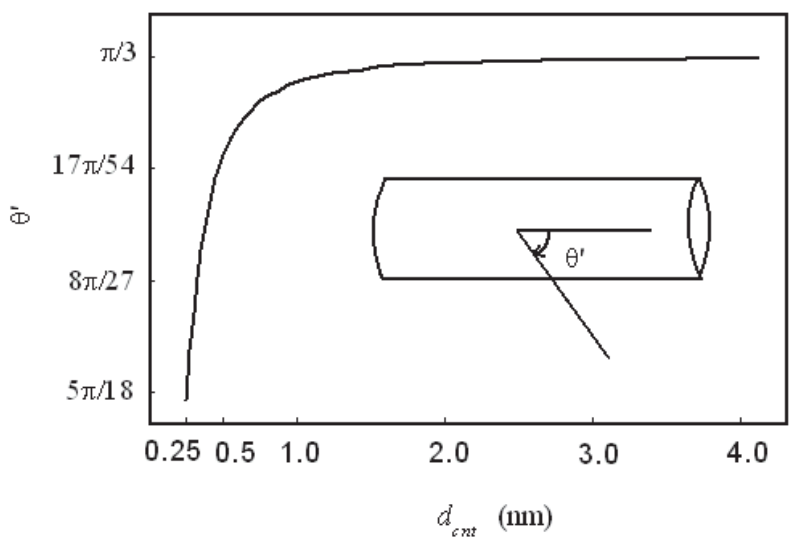

Fig. 18. Different C-C-C angles in zigzag SWCNTs with different diameters

The curve drawn from equation (63) is displayed in Fig. 18, which shows that the smaller the tube radius is the more sensitive the changing in C-C-C angle is. For the SWCNTs with diameter $0.4 \mathrm{~nm}, 1.0 \mathrm{~nm}, 2.0 \mathrm{~nm}$ and $4.0 \mathrm{~nm}$, the C-C-C angles in the tubes decrease $7.3 \%, 1.2 \%$, $0.3 \%$ and $0.08 \%$ from $120^{\circ}$ in the graphene.

\subsubsection{Armchair SWCNTs}

Taking the armchair SWCNTs as another example, we study the effect of the changing in diameter of SWCNTs on the value of C-C-C angle. The SWCNT structure and the geometric diagram are shown in Fig. 19, with which we can obtain,

$$
\begin{aligned}
& \tan \angle D B G=\frac{G D}{G B} \\
& \tan \angle E B H=\frac{H E}{H B}
\end{aligned}
$$

With $H E=G D$, the last equation becomes,

$$
\tan \angle E B H=\frac{H E}{H B}=\frac{G B}{H B} \cdot \tan \angle D B G \approx \frac{\sqrt{3}}{\cos \angle H B G}
$$

where the $\angle C B D$ does not change a lot when the graphene curls to the armchair SWCNT so that we make $\tan \angle D B G=\tan \frac{\angle C B D}{2} \approx \sqrt{3}$.

For an $(\mathrm{m}, \mathrm{m})$ armchair SWCNT, it has,

$$
\angle H B G=\frac{\pi}{2 m}
$$

The diameter of the tube is,

$$
d_{c n t}=0.0783 \cdot \sqrt{3} \mathrm{~m} \quad \mathrm{~nm}
$$




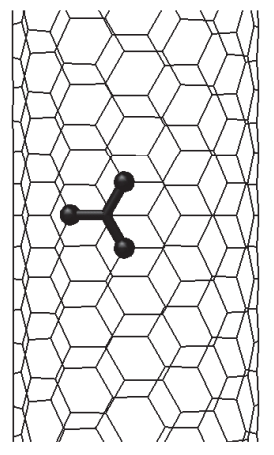

(a)
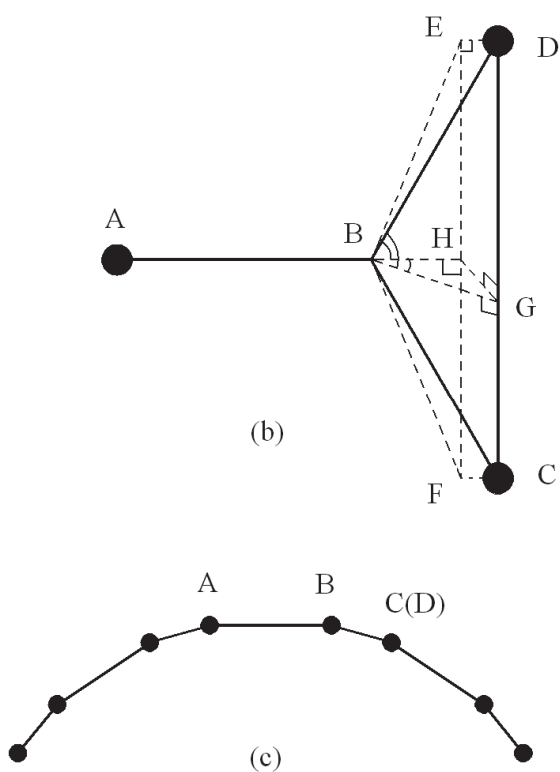

Fig. 19. C-C-C angles in the armchair SWCNTs

Substituting equation (67) and (66) into (65), we get,

$$
\tan \angle E B H=\frac{\sqrt{3}}{\cos \left(\frac{0.0783 \sqrt{3} \cdot \pi}{2 d_{c n t}}\right)}
$$

Thus, with the diameter of the armchair SWCNT provided, setting the AB direction in Fig. 19 as the direction of the 0 o fiber, we can calculate the C-C-C angles in armchair SWCNTs as,

$$
\theta^{\prime}=\operatorname{Arctan} \frac{\sqrt{3}}{\cos \left(\frac{0.0783 \sqrt{3} \cdot \pi}{2 d_{c n t}}\right)}
$$

The curve drawn from equation (69) is displayed in Fig. 20, which shows, as for the zigzag SWCNTs, that the smaller the tube radius is the more sensitive the changing in C-C-C angle is. For the armchair SWCNTs with diameter $0.4 \mathrm{~nm}, 1.0 \mathrm{~nm}, 2.0 \mathrm{~nm}$ and $4.0 \mathrm{~nm}$, the C-C-C angles in the tubes decrease $5.9 \%, 0.94 \%, 0.23 \%$ and $0.06 \%$ from 120 in the graphene. Comparing the armchair SWCNTs with the zigzag ones with same diameter, the changing in C-C-C angle in the armchair SWCNTs is smaller.

\subsection{Scale effect of elastic properties of SWCNTs}

With the usual continuum model of nanotubes, many researchers use the same isotropic material constants for CNTs with different radius so that the changes of the material properties due to changes in the radial size of CNTs can not be taken into account. In this 


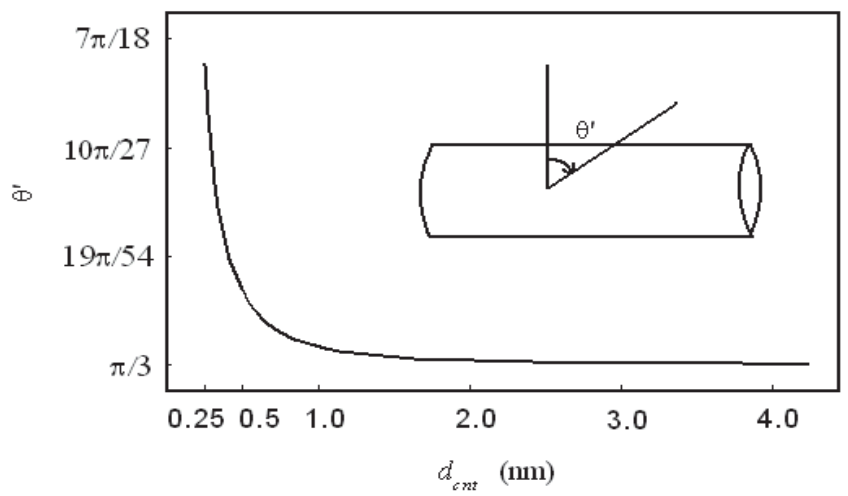

Fig. 20. Different C-C-C angles in armchair SWCNTs with different diameters

section, axial deformation of the SWCNTs with different diameter is analyzed with the finite element method to study the effect of the changes of the material properties for CNTs with different radius on the mechanical properties.

According to the theoretical model described above in this chapter, changes in the elastic properties of CNTs are mainly attributed to the changes of the C-C-C angles when to make CNTs by curling the graphene. The C-C-C angles in CNTs are related to the tube radius, while the corresponding $\mathrm{C}-\mathrm{C}-\mathrm{C}$ angles in the graphene induce varying degrees of anisotropy. Thus, we should choose different anisotropic material parameters for different radial size CNTs based on the parameters of the graphene with different C-C-C angles. Some elastic constants of graphite sheet and SWCNTs are listed in Table 3, the values for SWCNTs calculated with equation (57), (63) and (69). The subscript 1 and 2 in the table identify the along-axis direction of SWCNTs and the circumferential direction.

In the finite element simulation, the same axial strain is applied to each SWCNT, with one end of the tube fixed and at the other end imposed the axial deformation. The length of the tube is $6 \mathrm{~nm}$ and the axial compression strain is $5 \%$. Two series of the elasticity parameters, the isotropic ones (from equation (56)) and the anisotropic ones (from equation (57)), are both tried to obtain the axial forces in SWCNTs for comparison. With the results of the FEA, we use the following equation to define the scale effect of SWCNTs,

$$
\xi=\frac{R_{\mathrm{ANISO}}-R_{\mathrm{ISO}}}{R_{\mathrm{ISO}}}
$$

where $R_{\text {ANISO }}$ and $R_{I S O}$ are the axial forces obtained with the anisotropic parameters and the isotropic ones. The scale effect of SWCNTs with different radial size is shown in Fig. 21.

FEA results show that the anisotropy of the SWCNTs gradually increased with the decreases in the diameter, leading to more and more obvious scale effect. It can be seen from Fig. 21 that compared with the armchair SWCNTs the zigzag ones show more apparent scale effect and the scale effect of SWCNTs with diameter greater than $2 \mathrm{~nm}$ is negligible $(<0.05 \%)$, for tubes with any chirality. However, for zigzag SWCNTs with very small diameter, the scale effect could be very obvious up to $4.4 \%$. That is in good agreement with the other researcher's results. Thus, the scale effect should be considered in the accurate calculation about the mechanical behaviour of small SWCNTs. 


\begin{tabular}{|c|c|c|c|c|c|c|c|c|}
\hline \multicolumn{3}{|c|}{ C-C-C angles } & \multicolumn{5}{|c|}{ Mechanical parameters } & conclusion \\
\hline \multicolumn{3}{|c|}{ Graphene } & \multicolumn{5}{|c|}{$E=0.9755 \mathrm{TPa}, G=0.406 \mathrm{GPa}, v=0.201$} & Isotropic \\
\hline \multicolumn{3}{|c|}{ SWCNTs } & & & & & & \\
\hline & $\begin{array}{l}\text { Diameter } \\
(\mathrm{nm})\end{array}$ & $\begin{array}{c}\text { C-C-C } \\
\text { angle (o) }\end{array}$ & (1Рa) & (1Pa) & (1Рa) & & & \\
\hline \multirow{6}{*}{$\begin{array}{l}\text { Zig- } \\
\text { zag }\end{array}$} & 0.4 & 55.6298 & 1.00221 & 0.956849 & 0.403246 & 0.193494 & 0.202667 & Anisotropic \\
\hline & 0.6 & 57.9833 & 0.987757 & 0.967253 & 0.404666 & 0.197574 & 0.201762 & Anisotropic \\
\hline & 1.0 & 59.2586 & 0.980001 & 0.972556 & 0.405521 & 0.199891 & 0.201421 & Anisotropic \\
\hline & 2.0 & 59.8129 & 0.976654 & 0.974786 & 0.405908 & 0.200919 & 0.201304 & Isotropic \\
\hline & 3.0 & 59.9167 & 0.976029 & 0.975199 & 0.405982 & 0.201112 & 0.201284 & Isotropic \\
\hline & 4.0 & 59.9531 & 0.97581 & 0.975343 & 0.406008 & 0.20118 & 0.201277 & Isotropic \\
\hline \multirow{6}{*}{$\begin{array}{l}\text { Arm- } \\
\text { chair }\end{array}$} & 0.4 & 63.5548 & 0.988636 & 0.954613 & 0.40873 & 0.200935 & 0.208097 & Anisotropic \\
\hline & 0.6 & 61.5715 & 0.981558 & 0.966161 & 0.407195 & 0.201043 & 0.204247 & Anisotropic \\
\hline & 1.0 & 60.5640 & 0.977735 & 0.972147 & 0.406448 & 0.201172 & 0.202328 & Anisotropic \\
\hline & 2.0 & 60.1408 & 0.976084 & 0.974682 & 0.406142 & 0.201242 & 0.201532 & Isotropic \\
\hline & 3.0 & 60.0626 & 0.975776 & 0.975152 & 0.406086 & 0.201256 & 0.201385 & Isotropic \\
\hline & 4.0 & 60.0352 & 0.975667 & 0.975317 & 0.406066 & 0.201261 & 0.201334 & Isotropic \\
\hline
\end{tabular}

Table 3. Elastic constants of graphite sheet and SWCNTs 


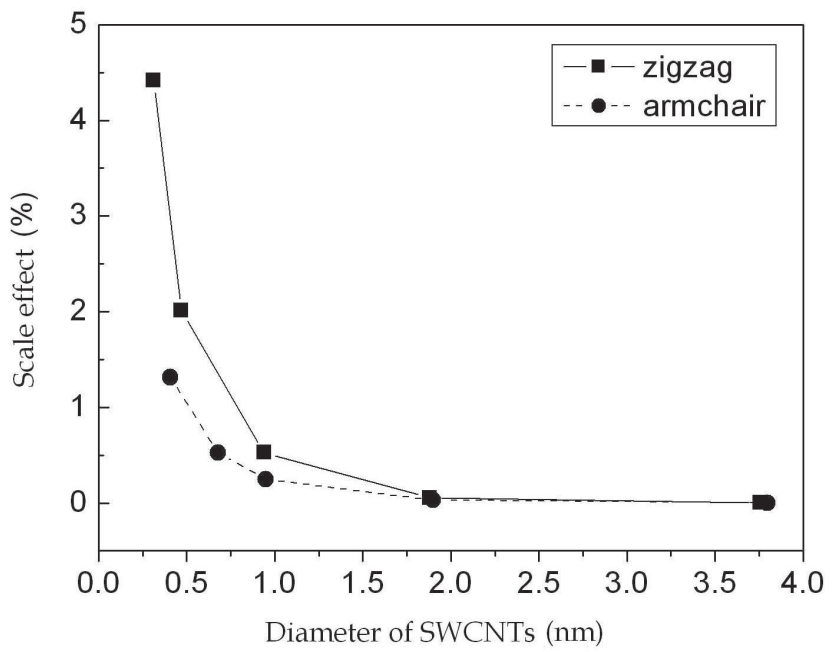

Fig. 21. Scale effect of SWCNTs with different diameters

With the data in Table 3 and the results from Fig. 21 we can see that, for SWCNTs with diameter greater than $2 \mathrm{~nm}$, the change of C-C-C angle due to the change in tube diameter is negligible so that the tubes could be treated as isotropic materials with the elastic parameters of graphene. For SWCNTs with diameter smaller than $1 \mathrm{~nm}$, the change of C-C-C angle due to the change in tube diameter should not be neglected and it is better for the tubes to be treated as anisotropic materials with the elastic parameters calculated from equation (57), (63) and (69).

\section{Conclusion}

A new equivalent continuum model is presented to theoretically investigate the elastic properties of the graphite sheet. By comparison, the equivalent model can properly reflect the actual elasticity status of graphite sheet. Further more this equivalent model is employed to study the radial scale effect of SWCNTs.

The C-C-C bond angles will change from the constant $120^{\circ}$ to smaller values when the graphite sheet curling to CNTs. The change of the C-C-C angle is obviously related to the change in CNT radius. Then the relationship between the anisotropy and the changes of the C-C-C angles of CNTs is deduced. The present theory not only clarify some puzzlement in the basic mechanical research of CNTs, but also lay the foundations for the application of continuum mechanics in the theoretical analysis of CNTs.

Based on above theory the scale effect of CNTs is studied. It is showed that the scale effect of the zigzag CNTs is more significant than the armchair ones. For SWCNTs with diameter greater than $2 \mathrm{~nm}$, the change of C-C-C angle due to the change in tube diameter is negligible so that the tubes could be treated as isotropic materials with the elastic parameters of graphene, and the scale effect could also be neglected no mater what chirality they are. However, for SWCNTs with diameter smaller than $1 \mathrm{~nm}$, the change of C-C-C angle due to the change in diameter should not be neglected (the scale effect neither) and it is better for 
the tubes to be treated as anisotropic materials with the elastic parameters calculated from corresponding equations.

It is theoretically demonstrated that the graphite sheet is in-plane isotropic under plane stress, which is mainly due to their special structure of C-C-C angles. Any deformation of the graphite molecule making changes in the $\mathrm{C}-\mathrm{C}-\mathrm{C}$ angles, e.g. curling, will introduce anisotropic elastic properties. That provides a direction for applying the composite mechanics to the research in the mechanical properties of CNTs, and also has laid an important foundation.

\section{Acknowledgment}

The authors wish to acknowledge the supports from the Natural Science Foundation of Guangdong Province (8151064101000002, 10151064101000062).

\section{References}

Brenner, D.W. (1990). Empirical potential for hydrocarbons for use in simulating the chemical vapor deposition of diamond films. Phys.Rev. B, Vol.42, No.15, (November 1990), pp. 9458-9471, ISSN 1098-0121

Gao; X.L. \& Li; K. (2003). Finite deformation continuum model for single-walled carbon nanotubes. International Journal of Solids and Structures, Vol.40, No.26, (December 2003), pp. 7329-7337, ISSN 0020-7683

Govindjee; S. \& Sackman; J. L. (1999). On the use of continuum mechanics to estimate the properties of nanotubes. Solid State Commun. Vol.110, No.4, (March 1999), pp. 227230, ISSN 0038-1098

Harik; V.M. (2001a). Ranges of applicability for the continuum-beam model in the mechanics of carbon-nanotubes and nanorods. Solid State Commun., Vol.120, No.7-8, (October 2001), pp. 331-335, ISSN 0038-1098

$\mathrm{Hu}$; N, Nunoya; K, Pan; D, Okabe; T. \& Fukunaga; H. (2007). Prediction of buckling characteristics of carbon nanotubes. International Journal of Solids and Structures, Vol.44, No.20, (October 2007), pp.6535-6550, ISSN 0020-7683

Jin; Y. \& Yuan; F.G. (2003). Simulation of elastic properties of single-walled carbon nanotubes. Compos. Sci. Technol., Vol.63, No. 11, (August 2003), pp. 1507-1515, ISSN 0266-3538

Krishnan; A., Dujardin; E., Ebbessen; T.W., Yianilos; P. N. \& Treacy; M. M. J. (1998). Young's modulus of single-walled nanotubes. Phys. Rev. B, Vol.58, No.20, (November 1998), pp. 14013 14019, ISSN 1098-0121

Li; C.Y. \& Chou; T.W. (2003). Elastic moduli of multi-walled carbon nanotubes and the effect of van der Waals forces. Compos. Sci. Technol., Vol.63, No.11, (August 2003), pp. 1517-1524, ISSN 0266-3538

Liu; J.Z., Zheng; Q.S. \& Jiang; Q. (2001). Effect of a Rippling Mode on Resonances of Carbon Nanotubes. Phys. Rev. Lett., Vol.86, No.21, (May 2001), pp. 4843-4846, ISSN 00319007

Lu; J.P. (1997). Elastic properties of carbon nanotubes and nanoropes. Phys. Rev. Lett., Vol.79, No.7, (August 1997), pp. 1297-1300, ISSN 0031-9007 
Mayo; S.L., Olafson; B.D. \& Goddard III; W.A. (1990). Dreiding: a generic force field for molecular simulations. J Phys Chem, Vol.94, No.26, (December 1990), pp. 8897-8909, ISSN 1932-7447

Poncharal; P., Wang; Z.L., Ugarte; D. \& W.A. de Heer. (1999). Electrostatic deflections and electromechanical resonances of carbon nanotubes. Science, Vol.283, No.5407, (May 1999), pp. 1513-1516, ISSN 0036-8075

Shen; H.S. (2004). Postbuckling prediction of double-walled carbon nanotubes under hydrostatic pressure. International Journal of Solids and Structures, Vol.41, No.9-10, (May 2004), pp. 2643-2657, ISSN 0020-7683

Sudak; L.J. (2003). Column buckling of multiwalled carbon nanotubes using nonlocal continuum mechanics. J. Appl. Phys., Vol.94, No.11, (November 2003), pp. 72817287, ISSN 0021-8979

Wang; C.Y., Ru; C.Q. \& Mioduchowski; A. (2003a). Axially compressed buckling of pressured multiwall carbon nanotubes. International Journal of Solids and Structures, Vol.40, No.5, (July 2003), pp. 3893-3911, ISSN 0020-7683

Wang; Q., Varadan; V.K. \& Quek; S.T. (2006). Small scale effect on elastic buckling of carbon nanotubes with nonlocal continuum models. Physics Letters A, Vol.357, No.2, (September 2006), pp. 130-135, ISSN 0375-9601

Wang; Q. \& Varadan; V.K. (2007). Application of nanlocal ealstic shell theory in wave propagation analysis of carbon nanotubes. Smart Mater. Struct., Vol.6, No.1 (February 2007), pp. 178-190, ISSN

Xin; H., Han; Q. \& Yao; X.H. (2007). Buckling and axially compressive properties of perfect and defective single-walled carbon nanotubes. Carbon, Vol.45, No.13, (November 2007), pp. 2486-2495, ISSN 0008-6223

Xin; H., Han; Q. \& Yao; X.H. (2008). Buckling of defective single-walled and doublewalled carbon nanotubes under axial compression by molecular dynamics simulation. Compos. Sci. Technol., Vol.68, No.7-8, (June 2008), pp. 1809-1814, ISSN 0266-3538

Yang; X.G., \& Zeng; P. (2006). Numerical simulation of anisotropic mechanical Properties of nano-graphite crystals. Journal of basic science and engineering, Vol.14, No.3 (Sep tember 2006), pp. 375-383, ISSN 1005-0930

Yao; X.H. \& Han; Q. (2007). Postbuckling prediction of double-walled carbon nanotube under axial compression. Eur. J. Mech .A-Solid, Vol.26, No.1, (January-February 2007), pp. 20-32, ISSN 0997-7538

Yao; X.H. \& Han; Q. (2008). Torsional buckling and postbuckling equilibrium path of double-walled carbon nanotubes. Compos. Sci. Technol., Vol.68, No.1, (January 2008), pp. 113-120, ISSN 0266-3538

Yao; X.H., Han; Q. \& Xin; H. (2008). Bending buckling behaviors of single- and multi-walled carbon nanotubes. Computational Materials Science, Vol.43, No.4, (October 2008), pp. 579-590, ISSN 0927-0256

Zhang; Y.Q., Liu; G.R. \& Xie; X.Y. (2005). Free transverse vibrations of double-walled carbon nanotubes using a theory of nonlocal elasticity. Phys. Rev. B, Vol.71, (May 2005), pp. 195404:1-7, ISSN 1098-0121 
Yang; X.G., \& Zeng; P. (2006). Numerical simulation of anisotropic mechanical Properties of nano-graphite crystals. Journal of basic science and engineering, Vol.14, No.3 (Sep tember 2006), pp. 375-383, ISSN 1005-0930 


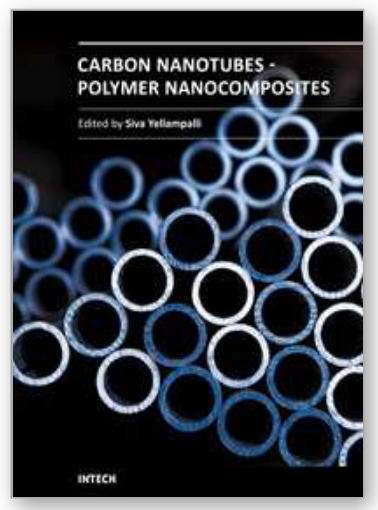

\section{Carbon Nanotubes - Polymer Nanocomposites}

Edited by Dr. Siva Yellampalli

ISBN 978-953-307-498-6

Hard cover, 396 pages

Publisher InTech

Published online 17, August, 2011

Published in print edition August, 2011

Polymer nanocomposites are a class of material with a great deal of promise for potential applications in various industries ranging from construction to aerospace. The main difference between polymeric nanocomposites and conventional composites is the filler that is being used for reinforcement. In the nanocomposites the reinforcement is on the order of nanometer that leads to a very different final macroscopic property. Due to this unique feature polymeric nanocomposites have been studied exclusively in the last decade using various nanofillers such as minerals, sheets or fibers. This books focuses on the preparation and property analysis of polymer nanocomposites with CNTs (fibers) as nano fillers. The book has been divided into three sections. The first section deals with fabrication and property analysis of new carbon nanotube structures. The second section deals with preparation and characterization of polymer composites with CNTs followed by the various applications of polymers with CNTs in the third section.

\section{How to reference}

In order to correctly reference this scholarly work, feel free to copy and paste the following:

Qiang Han and Hao Xin (2011). Elastic Properties of Carbon Nanotubes, Carbon Nanotubes - Polymer Nanocomposites, Dr. Siva Yellampalli (Ed.), ISBN: 978-953-307-498-6, InTech, Available from: http://www.intechopen.com/books/carbon-nanotubes-polymer-nanocomposites/elastic-properties-of-carbonnanotubes

\section{INTECH}

open science | open minds

\section{InTech Europe}

University Campus STeP Ri

Slavka Krautzeka 83/A

51000 Rijeka, Croatia

Phone: +385 (51) 770447

Fax: +385 (51) 686166

www.intechopen.com

\section{InTech China}

Unit 405, Office Block, Hotel Equatorial Shanghai

No.65, Yan An Road (West), Shanghai, 200040, China

中国上海市延安西路65号上海国际贵都大饭店办公楼 405 单元

Phone: +86-21-62489820

Fax: $+86-21-62489821$ 
(C) 2011 The Author(s). Licensee IntechOpen. This chapter is distributed under the terms of the Creative Commons Attribution-NonCommercialShareAlike-3.0 License, which permits use, distribution and reproduction for non-commercial purposes, provided the original is properly cited and derivative works building on this content are distributed under the same license. 